OSTEOMA OF THE ORBIT, REMOVAL WITH PRESERVATION OF VISION.

Read before the Section of Ophthalmology, at the Forty third An nual Meeting of the American Medical Association, held at Detroit. Mich., June, 1892.

BY EDWARD JACKSON, M.D

PROFESSOR OF DISEASES OF THE EYE IN THE PHILADELPHA POLYCLINIC, SURGEON TO THE WILLS EYE HOSPITAL.

The following case seems to me to be of sufficient importance to place upon record:

Miss M., æt. 18, had noticed two years before, a swelling of the left upper lid, pretty uniform and unaccompanied by pain or soreness. This swelling has since then increased gradually until the present time. For the last few months, there has been some pain over the upper inner angle of the left orbit.

About one year ago she noticed that the vision in the left eye was not so good as in the right, but the eyes had not previously been separately tested, and there has been no deterioration of vision since. In the right eye the vision is as good as it has ever been. In the right eye the media are clear; there is hyperopic astigmatism; the disk is oval clear, of good color; fundus dark, normal. Left eye, media clear; higher hyperopic astigmatism; disk oval, clear, of good color; arteries bear due proportion to veins in size. Muscle tests, show right hyperphoria of from 0 to 1 centrad laterally there is muscular balance, power of abduction 5 centrads; adduction 10 to 12 centrads ; sursumduction, right or left, 1 centrad. A hard tumor is felt below the upper margin of the left orbit from which it is separated by a distinct though narrow sulcus. It extends from the inner third to the outer angle of the orbit, and about one centimetre in the vertical direction. The left eye is displaced downward one centimetre and forward eight millimetres. No lateral displacement can be recognized

1891, October 9, I removed the tumor. A free incision was made through the upper lid just below and parallel to the upper margin of the orbit, and the growth freely exposed by the retraction of all the soft parts; it proved to be entirely hard and connected with the roof of the orbit by an apparently broad base and to extend more than half way back to the apex of the orbit. The exposed surface was bitten away with rongeur forceps. But it was soon evident that these would remove but a small part of the growth. The saw was tried but could not be used to advantage. The chisel gave no result, because I feared to apply sufficient force, not knowing at what point it might cause fracture. With a hand drill however, three holes were made in different directions, and the intervening bone split with the chisel, separating the growth from its attachments, and dividing it in two principal fragments which were then extracted. During this process, there being a tendency to extrusion of the globe between the lids, at the suggestion of Dr. Risley, these were stitched together and the stitch removed at the completion of the operation. The attachment to the walls of the orbit proved to be upward and inward three-fourths of an inch back from the orbital margin, and the removal of the tumor opened the ethmoidal sinuses so that blood escaped through the nose. The tumor weighed about 13 grams. The wound was washed out with bichloride solution, a catgut drain brought out at the outer angle, and the wound closed with seven sutures a gauze dressing was applied with a bandage, so as to make firm pressure on the lower lid. The operation was done under ether and lasted one hour and three quarters.

The temperature did not rise above the normal, the wound closed by first intention. Drainage and sutures were removed on the seventh day. At the first dressing, next morning, there was found considerable swelling, especially of the conjunctiva which protruded between the lids, and could not be entirely returned. Next day, the protruding portion was snipped off, after which the swelling and hyperæmia rapidly diminished. At the end of two weeks the swelling was greatly reduced and the globe had almost, but not entirely returned to its normal position. There was, however, very little power of moving the eyeball, and diplopia was experienced in all parts of the visual field except the centre. There was complete ptosis.

From this time there was rapid improvement in the motility of the globe, and at the end of four weeks she experienced diplopia only in the extreme periphery of the field of binocular vision. The ptosis had decidedly lessened. One month later, her recovery was complete except a moderate ptosis. Her condition continued the same up to the time she was last heard from. With Right $+0.25 \mathrm{sph}$. $\subsetneq+2$. cyl ax. $110^{\circ}, \mathrm{V}=4+4$ mostly. Left $+0.25 \mathrm{sph} . \leftrightharpoons+3$. cyl. ax. $65^{\circ}$, $=4-15$, about the same as before the operation.

It is interesting to note in this case that although the tumor appeared in the outer two-thirds of the orbit, its origin was from the upper inner angle about three-fourths of an inch back from the orbital margin, and the displacement was directly forward and downward.

Although the eye-ball had been carried forward to the extent of almost a centimetre and the insertions of the muscles to that extent removed, there had been no squint or diplopia, there was very little heterophoria, and no marked weakness of the ocular movements.

All the movements of the eyes except the elevation of the upper lid were completely restored within a few weeks.

In the removal of the exostosis which was throughout almost its whole extent, of ivory like hardness, the common bone drill worked simply by hand, penetrated rather rapidly and proved by far the most efficient as well as the safest means of attack.

\section{A CASE OF RARE FORM OF ORBITAL TUMOR.}

Read in the Section of Ophthalmology at the Forty-third annual meeting of the American Medical Association, held at Detroit, Mich. June, 1892.

BY G. E. FROTHINGHAM, M.D., OF DETROIT, MICH.

Cases in which tumors are developed in both orbits are extremely rare. That the tumors should occupy exactly the same position in each orbit, and correspond in form and size, and develop at nearly the same time, is still more remarkable. For these reasons, as well as some other unusual characters, I have thought the following case worthy to be put on record.

Miss E. D., age sixteen, of Canton, Michigan, consulted me January 11,1892

The father gave the following history: In May, 1890, she noticed a tumor of the left orbit. There was no pain in the tumor or protrusion of the eyeball, but the tumor projected somewhat, and was situated above the eye, extending from the inner extremity of the superciliary ridge to the outer canthus, and causing a prominence parallel with, and just below this bony arch. The tumor felt hard and was slightly movable on firm pressure. She visited the clinic at Ann Arbor, and had an operation for its removal, in December of the same year. A large firm tumor was removed. The eyeball protruded after the operation, presenting the appearance shown in the photograph here presented which was taken on the 10th of IIay last. The recovery from the operation was rapid, the patient leaving the hospital in about a week after it was performed. While recovering from the effect of the operation, she was feeling about her right eye, and noticed a tumor over it in the same position as the one which had been removed from the left orbit. It had also the same feeling and was slightly movable; she also noticed before she left the hospital that there was a small tumor at the outer portion of the left orbit and also ne near the center of the lower portion below the eyeball

At the time I examined her, there was a large tumor of the right orbit, causing a prominence projecting to about the same level with the bony margin, and about an inch and a half in length on its anterior border. It was situated above the levator palpebrarum and close to the orbital plate of the frontal bone. The eyeball was displaced downward and there was double vision, except when looking sharply downward, the image of the right eye being above that of the left. There was a tumor at the outer margin of left orbit, slightly movable and apparently extending deeply inward. There was also a small, very movable tumor below the left eye, which still remains. On the 14 th of January, assisted by my son, Dr. G. E. Frothingham, Jr., 
and by Dr. L. Connor, I operated for the removal of the tumor of the right orbit.

An incision was made along its anterior border down to the tumor, which was carefully dissected from the surrounding tissues, by means of the handle of the scalpel and probe pointed seissors. When this had been done, the tumor was carefully dragged from its bed, bringing with it the firmest strands of connective tissue, which extended inward from its deep border at the bottom of the orbital cavity. The supra-orbital nerve was so intimately blended with the tumor, that it had to be sacrificed. After the hremorrhage had ceased, a small drainage tube was inserted sufficiently deep to allow escape of any blood that might accumulate from subsequent oozing, and the edges of the cut then brought together by suture, and the wound dressed with cotton saturated in solution of bichloride of mercury, 1-2000. There was but little reaction, and the patient left the hospital in one week from the operation, with the wound healed and the parts almost entirely free from swelling. The double vision was completely relieved by the removal of the tumor. The tumor measured a little more than an inch and a half along its anterior border, and about the same along its posterior border. It extended also a little more than an inch and a half into the orbit. It was wedgeshaped, the base forming the anterior portion of the tumor. The thickness of the firm portion was, at the base, a little more than three-eighths of an inch at the inner extremity, and slightly over one-fourth of an inch at the outer extremity. It grows gradually thinner, as it approaches the edge.

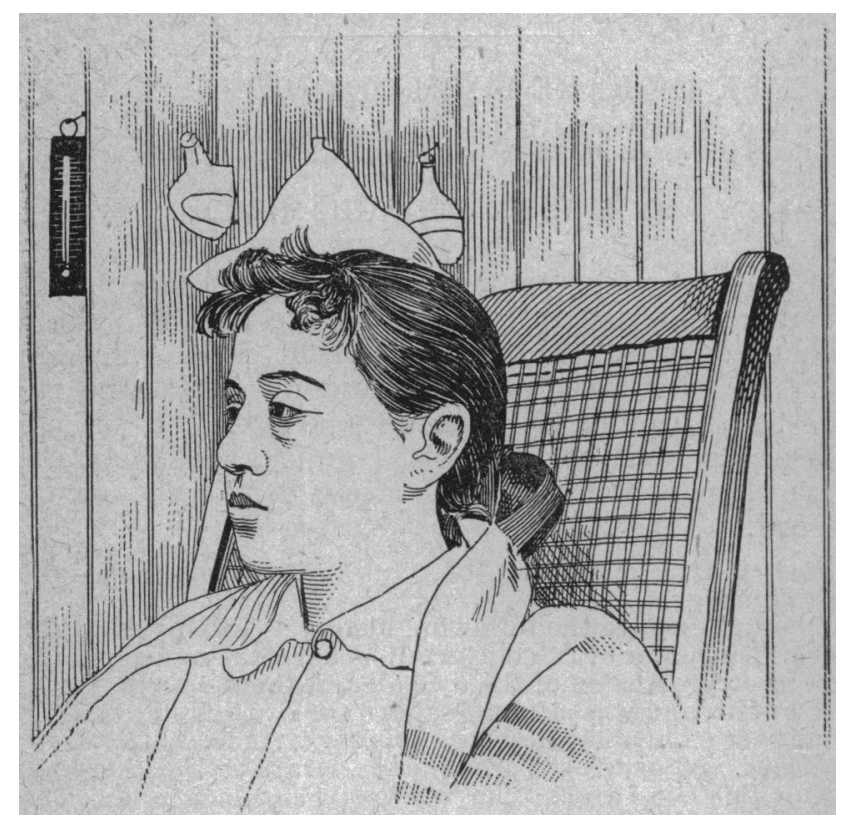

It is very firm to the feeling like fibro-cartilage, and resembles on section the appearance of dense, white fibrous tissue, or condensed connective tissue.

There was some degree of ptosis of the lid, after the operation, but that was soon recovered from. There has been no sign of recurrence of the tumor of the right eye up to the present time. There is no resulting exophthalmus, and vision is unimpaired. The patient being anxious to be relieved from the proptosis of left eye, I operated upon it on the 10th of May, last Making an incision, extending the outer canthus, and then dividing the palpebral ligaments of both lids to a sufficient extent. I thus made an opening sufficiently large, without endangering the nerve supply of the orbicularis. Through this opening I removed a tumor about three-fourths of an inch long, half an inch wide, and one-eighth of an inch thick, extending into the orbit behind the globe. The periosteum seemed thickened at the bottom of the orbit, but no distinct tumor could be felt. The tumor below the eye could not be removed through this opening, and I thought it more judicious to leave it for a subsequent operation, than to make so extensive dissections at one time. The wound was treated as in the operation upon the right eye. The inflammatory reaction was, however, much greater, and seemed quite threatening during the first thirty-six hours. It then began to subside. The conjunction was much swollen, however, and it was two weeks before it could be fully replaced under the lower lid. The vision is unimpaired and the proptosis seems less though not greatly diminished by the operation.

An examination made by Dr. George Duffield, shows the tumor to be a fibro-sarcoma, with the fibrous element very largely predominating-a tumor that is sometimes called a fibroma. It seems to develop from connective tissue, and is not a frequent form of sarcomatous tumors. It is said that a preponderance of cellular elements is the peculiar feature of all sarcomata. At any rate, those I have found, either within the eye or the orbit, were rich in cells and quite friable, instead of being firm and tough like the one here presented. I believe such tumors nearly always become very rich in cells, and have blood-vessels within them before they have grown to the size this one had reached.

The tumor removed from the left orbit by Prof. Carrow, Dec. 4, 1890, was reported by the pathologist as a sarcoma, but the variety was not given.

\section{Discussion.}

Dr. H. Knapp, New York:-I beg to make some remarks especially with reference to the paper of Dr. Frothingham. I wish to point out the difficulty in diagnosis in some of these cases, as on three oceasions I have fallen into a great mistake. Some of these tumors are benign although they present the features of malignancy. I remember three cases of this kind. The first, was an individual about 20 jears of age, with a tumor situated deeply in the orbit producing exophthalmus. It was supposed to be a sarcoma and yet it completely disappeared.

In the second case, there was considerable exophthalmus from large growths in different parts of the orbit. The eye was taken out by one of the surgeons of the Manhattan Eye and Ear Hospital and the tumor cleanly removed. On microscopic examination. it was pronounced to be a smallcelled sarcoma. The patient came to me two years later with the same condition in the other eye: There was great protrusion of the eye and solid masses were distinctly felt in the orbit. I could not advise the woman to have that eye removed and $I$ was not sure that otherwise the radical removal of these tumors could be accomplished. She was under general treatment and three or four months afterward I heard that she was better and that the tumors gradually diminished in size. After about 15 months she again came to me and was perfectly cured. The growths and the exophthalmus had disappeared without leaving a trace and without any injury to the functions of the eye.

Another case was sent to me last year, by Dr. Morgan, of Springfield,Massachusetts. The tumor had sprung from the inner side of the orbit and seemed to be connected with the periosteum. It looked like a periosteal sarcoma. Dr. Morgan removed the growth and the microscope showed that it was sarcoma. That patient had similar growths in the nasal passage of the same side. It seemed to be a case of sarcomatous growths of the nasal passages protruding into the orbital cavity. After the removal by Dr. Morgan, there was a rapid relapse. In several months the growth was as large as before. The tumor seemed to go into the wall of the orbit so deeply that $I$ did not think any operation could be done with benefit. The orbital small celled sarcomata are as malignant as any tumors can be. This patient after leaving me went to an electrician and also took some indifferent remedies. She perfectly recovered. She said that the improvement began after being under the electric treatment for four or six weeks and had steadily continued. Not long ago she presented herself to me and there was no trace of the trouble left. The orbit was normal and the nasal passages were free. She came to express her gratitude that $I$ had advised her not to be operated upon.

What are these growths that come under the mask of sarcoma and evidently are not malignant? They are not fibromata for they do not disappear. The only thing that 1 can imagine is that they are lymphomata. In the first case the diagnosis of lymphoma was not so difficult because there were swellings in other parts. It was a symptom of Hodgkin's disease. In the other two cases, there was nothing to lead me to such a diagnosis. The disease was purely local. The histology of the tumor was in all the same, a small celled tumor with more or less fibrous tissue. When 
I see such a tumor, if I am sure that it is not in connection with the orbital walls, I hesitate to advise its immediate removal. If it progresses there is nothing else to be done. If the tumors are multiple and malignant, we cannot do any good. If there is such doubt and I think the doubt cannot be entirely excluded, I would rather wait before committing myself to the removal of the eye or tumor.

The different elements which constitute these tumors may perhaps lead to the diagnosis of lymphoma. We must also bear in mind the possibility of diffuse or disseminate sarcoma which is the worst kind of all. We think to have removed them cleanly and it is not two months before you see tumors in the neighborhood. Some of these cases have been sent to me for operation after some of the tumors have been removed. I have removed the orbit's contents cleanly to the periosteum and on examining them I have found all through the orbital tissue, patches and nodules of sarcomatous tissue. In one case the diffuse sarcoma was melanotic and had invaded the optic nerve. These points are to be taken into consideration in the diagnosis of these tumors of which some are certainly benign.

Dr. Reeves, Toronto:-Seventeen years ago I enucleated an eye for sarcoma in which there was found a melanotic tumor. The tumor was not examined microscopically. It was a firm tumor occupying a large portion of the vitreous chamber. About sixteen years later, two years ago, the patient again, presented himself. For fifteen years there had been no sign of disease of the orbit. On examination I found indications of sarcoma of the orbit and two years ago last October, I operated. The patient had marked cephalalgia. I cleaned out the orbit completely and applied chloride of zinc. Microscopical examination showed the tumor to be a melanotic sarcoma of the optic nerve. The patient is in good health with no sign of return of the growth. The cephalalgia disappeared in a few weeks after operation.

do not consider this a case of true recurrence of sarcoma, I do not believe that sarcoma can remain latent for fifteen years. It is a case rather that may be explained on the principles of the abnormal development of embryonic tissue which existed there from the beginning.

Dr. Julian J. Chisolm, Baltimore :-About a month ago, 1 had occasion to take out an eye in a case that presented the question, at what period during the existence of an intraocular tumor is systemic infection established? The patient had bad detachment of the retina with absolute loss of vision, for one year but unaccompanied by pain. A week before I saw him the first pain was experienced. The eye was much injected, there was the usual tension, and the pupil was slightly dilated. These were all evidences of an intra-ocular growth and a correct diagnosis was made although the detachment of the retina excluded the use of the ophthalmoscope. When the eye was removed and opened, I found a growth not larger than a pea which proved itself a melanotic sarcoma. It had infiltrated the sclerotic although the inflammatory symptoms with tension had existed only one week and the tumor did not occupy one-sixth of the vitreous space.

Dr. S. C. Ayers, Cincinnati :-I have recently had a case of tumor of the orbit, which was to me, one of extreme interest. I first saw the patient a year ago last March. There was a little exophthalmos and vision was considerably reduced. There was infiltration of the optic nerve and under the lower lid one could feel a suspicion of a growth. I again saw the patient a week or ten days later. Vision had fallen to one-half and I thought that I could feel that the tumor had developed in these few days. I gave my opinion that it was a sarcoma originating in the apex and pressing forward rapidly and that vision would be more and more reduced and advised removal of the tumor and removal of the globe if necessary. I thought that it was not possible to save the globe. I saw the patient no more until fall. He went elsewhere and an attempt was made to remove the tumor and save the globe, but the attempt was not successful in either direction. Panophthalmitis followed the operation and the man suffered intensely for a long time and the tumor again began to grow. When I saw him in the fall,there were four or five large firm nodules around the rim of the orbit. I said then that it was useless to do anything. I saw the patient from time to time, but the tumor was evidently not growing forward, but was clearly advancing toward the brain. He was soon bed fast and his nutrition seriously impaired. He lost flesh and in February had paralysis of the right arm. The tumor involved the right eye. He died about six weeks ago. During the latter part of his illness there was observed a ridge or elevation in the periosteum extending upwards and following the suture of the parietal bone. The bone was tender. $\mathrm{He}$ also complained a good deal of pain in the region of the cerebellum. When the post-mortem was made we found that the parietal bone and the upper portion of the frontal and of the occipital bones were softened. The parietal was so softened that it could almost be penetrated with a knife. The internal surface of the parietal was covered with a growth looking like granulations. The internal and external surface of the dura was covered with a similar deposit. The granulations on the internal surface were larger and more exuberant than those in other places. The sphenoid bone could be cut with a knife. The tumor had not involved the brain, but had simply grown over the anterior surface and pressed it down. The operation originally would have had no more influence than to prolong life for a little while. The extension of the growth backwards and the involvement of the bone and dura mater was something new in $\mathrm{my}$ experience.

Dr. Johnson, Paterson, N. J.:-I agree with Dr. Knapp as to the necessity of considering carefully the desirability of making these operations, on account of the possibility that the tumors may be benign in their nature. At the same time I had occasion to see a month or two ago a case in which, after operation was advised, delay had been made as a result of the desire of the patient's family. When the tumor became sufficiently increased in size to alarm them, they permitted operation, which was followed in a short time by rapid recurrence of the tumor and death of the patient. I think that it is desirable, if operations on orbital tumors are to be made, that they should be made early. I have operated on a number of orbital tumors, some of which were sarcomatous in their nature; one in particular, on which I operated eight or nine years ago, was reported in the Archives of Ophthalmology. The growth was examined microscopically by Dr. Prudden, and pronounced to be a myxosarcoma of the optic nerve. Up to this time there has been no recurrence. Whether or not this was one of those tumors with a malignant appearance and a benign effect, I cannot say. In these tumors presenting a malignant appearance it is desirable that they should be removed as soon as we can make up our minds whether they are malignant or benign while it is still possible to remove the entire growth.

Dr. J. A. White, Richmond, Va. :-I have seen cases of sarcoma which did not recur. Fibroma is not in its nature malignant, but I consider it more malignant than ordinary sarcomatous growths, because it almost invariably recurs. I have seen only three cases of fibroma of the orbit, and in every case there was recurrence. I think that it is difficult to state how much the fibrous change has taken place in certain tissues, because of the little difference microscopically from the normal appearance.

In regard to whether or not the last growth will recur, I do not know, because I have never seen a case of miliary tuberculosis of the orbit before.

Dr. George E. Frothingham, Detroit :-I should like to add one word in connection with these tumors. It has been questioned here whether a melanotic sarcoma can remain stationary as long as fifteen years. I remember a case where a melanotic sarcoma of the choroid had ruptured through the external tunics. It was thoroughly removed, but after six or eight years, it recurred. All the contents of the orbit were then removed, and after four or five years, it again returned.

As to the question of the malignancy of these growths, $\Upsilon$ think that we have very imperfect knowledge. One fact has. been well established, that is that some of these cases will never recur, while in others operation seems to stimulate their growth. In 1876 , I had a peculiar case which bears upon this matter. A patient came with a painful, blind eye, the lens opaque and the iris attached to the lens, and the tension increased. He had such severe pain that enucleation was decided upon. Fearing malignancy, the nerve was cut as far back as possible. After removing the tumor, I examined the eye, and found about the optic nerve some connective tissue which felt a little harder than normal. Internal examination showed no tumor within the eye. It was simply a case of chronic inflammatory disease. A few months afterward the patient came with a large mass protruding from the orbit. I think that within five months after the eye had been removed, that immense mass developed. It was so evidently malignant that $I$ at once decided to operate. The whole contents of the orbit were removed. The growth was so closely connected with the bony wall of the orbit that I decided to apply chloride of zinc paste. This produced necrosis of the bony wall, so that the brain protruded into the orbit, and then the wound granulated. 
His physician reported a few months later that he had died with all the evidences of malignant disease of the kidney. Examination showed the growth to be a giant-celled, rapidly growing sarcoma.

Another case occurred in a child 2 years of age. A firm tumor was found at the outer part of the orbit. It had the feel of a sarcoma, and connective tissue was found extending into the bone. It was cut loose, but rapidly recurred, and the child died.

\section{THE ETIOLOGICAI, RELATION OF NASAI, DISEASES TO AFFECTIONS OF THE EYES.}

Reard in the Section of Ophthalmology at the Forty-third Annual Meeting of the Anteriean Medical Association, held at Detroit, itich., June 1892 .

\section{BY DR. H. GRADLE,} CHICAGO.

Within the past eight years various publications have appeared intended to show a connection between certain ocular and nasai disturbances.

The proof that lesions of the orbit, eye and its appendages or symptoms referable to these parts are induced by preëxisting nasal diseases, can be furnished in various ways, viz.:

1. We may trace anatomically the extension of disease of the nasal cavity into the orbit or into the appendages of the eye.

2. We may observe clinically the involvement of these parts or of the eye itself in disease consecutive to and in direct connection with nasal disease.

3. We may influence in a characteristic manner by means of nasal treatment eye effections the prognosis of which is definitely known.

4. More decisive than these methods is the experimental production of eye lesions in animals in consequence of nasal disturbances. As yet, nothing has been done in this line. But practically equivalent to experiments on animals are various observations of disturbances in the eyes or lids following experimental irritation or irritant treatment of the nose in patients.

Of these different modes of proving the connection between ocular and nasal diseases the first method is the most definite one, no matter whether we trace the extension of the disease during life or at an autopsy. The second way, the clinical observation of the evolution of the ocular disorder demands reliance upon the patient's statements, since the physician can but rarely see such cases at their incipiency. The sequence of the two disorders is after all only suggestive of the dependence of one upon the other; it is not an absolute proof. It is only the repeated observation of cases with similar histories in this respect that can establish the etiological relationship with some certainty. But it must not be demanded that because a certain eye disease is of nasal origin in some instances it should always depend upon the same etiological conditions.

Most of the writers on this subject have employed the third mode of reasoning - that is to say, the therapeutic test.

When they observed an improvement in certain eye symptoms after nasal treatment they reasoned that the nasal condition has been the cause producing them.

This mode of reasoning however, is beset with errors, against which we must guard ourselves. The prognosis is not always so certain in the class of diseases principally referred to, especially asthenopic complaints, so that an improvement following any given treatment can be attributed to that treatment only if it sets in very promptly. We must be sure that neither other measures employed, nor the mere lapse of time are responsible for the patient's improvement.

Moreover, in estimating the beneficial results of any new treatment, particularly of a surgical character, upon nervous symptoms we must not ignore the possibility of mental suggestion. For these reasons as well as on account of the short period of their observation some of the cases and conclusions recorded in literature are not as convincing of the relation of the nose to the eye, as their authors claim.

When the question arises whether actual ocular lesions and not mere functional derangements are to be considered as secondary results of nasal disease the therapeutic test cannot be regarded as an absolute criterion. For lesions once started do not necessarily cease on removal of the conditions that induced them. In such instances the etiology can only be established on the basis of careful histories.

American authors have mostly dealt with functional derangements only, but cases published by Gordon, de Schweinitz and various European observers, as well as my personal experience, point out that also different lesions of the eye and its appendages may be of nasal origin.

On the basis of personal experience, and aided by the published observations of others, I shall attempt to classify the various ocular derangements dependent upon nasal disturbances, and will aim to distinguish critically between actual proof of such connection and mere probability of nasal origin.

I. In diseases of the lachrymal passages nasal affections are found present in the majority of instances [ [2" *2: to and personal experience] + . In speaking of nasal diseases or anomalies, I refer to demonstrable lesions or marked deviations from the normal shape, no matter whether they give the patient annoyance or not. If we were to diagnosticate nasal disorders only on the strength of the patient's statement as to the discomfort in the nose, we we would overlook many instances of chronic disorders in the less observing class of people. On the other hand, whenever I speak of acute nasal catarrh, I do not rely on the patient's reference to a "cold," but demand a definite history of the symptoms of coryza.

The mere coincidence of nasal disorders, so common in our climate, with lachrymal disease, is far from being a proof of their correlation. Stronger, however, is the evidence that in one-sided lachrymal disease the affected passage corresponds almost invariably to the more abnormal side of the nose. A direct proof that the nose is the starting point of the disease of the tear-canal can only be obtained when an intelligent patient observes the insufficiency of the tear duct or the inflammation of the sac beginning during an acute attack of nasal disease. Of such instances I have a number in my records. I do not wish to overlook, however, that occasionally lachrymal disease, especially narrowing of the canaliculi, is the result of conjunctival disease.

The therapeutic test is not decisive in lachrymal affections. Neither in stricture of the duct nor in suppuration of the sac, can removal of the nasal lesions restore the integrity of the lachrymal passage 